\title{
Minimizing topological entropy for maps of the circle
}

\author{
LOUIS BLOCK, ETHAN M. COVEN† AND ZBIGNIEW NITECKI \\ From the University of Florida, Gainesville, Fla., Wesleyan University, Middletown, \\ Conn., and Tufts University, Medford, Mass.
}

(Received 10 December 1980)

Abstract. For each $n \geq 2$, we find the minimum value of the topological entropies of all continuous self-maps of the circle having a fixed point and a point of least period $n$, and we exhibit a map with this minimal entropy.

\section{Introduction}

This paper is concerned with the following problem.

For each $n \geq 2$, find a continuous map $f_{n}$ of the circle to itself, having a fixed point and a point of least period $n$, minimal in the sense that ent $\left(f_{n}\right) \leq \operatorname{ent}(f)$ for every continuous map $f$ of the circle having a fixed point and a point of least period $n$. Determine ent $\left(f_{n}\right)$.

Here ent $(\cdot)$ denotes topological entropy.

The solution to the analogous entropy-minimizing problem for maps of the interval was discovered in the course of investigations having to do with Šarkovskii's theorem. Recall the Šarkovskii ordering $\triangleleft$ of the positive integers

$$
3 \triangleleft 5 \triangleleft \cdots \triangleleft 2 \cdot 3 \triangleleft 2 \cdot 5 \triangleleft \cdots \triangleleft 2^{2} \cdot 3 \triangleleft 2^{2} \cdot 5 \triangleleft \cdots \triangleleft 2^{2} \Delta 2^{1} \triangleleft 2^{0} \text {. }
$$

Šarkovskii's theorem [4], [5], [3] states that, if a continuous map of a compact interval to itself (or to the reals) has a point of least period $n$, then it has a point of least period $m$ for every $m \triangleright n$.

P. Štefan [5] has described a set of constructions which, for each $n \geq 2$, yields a map $g_{n}$ of the interval having a point of least period $n$ but no point of least period $m$ for any $m \triangleleft n$. It turns out [5], [3] that the maps $g_{n}$ are the solution to the entropy-minimizing problem for maps of the interval: ent $\left(g_{n}\right) \leq$ ent $(g)$ for every continuous map $g$ of the interval having a point of least period $n$. (If $g$ maps a compact interval $I$ into the reals, then ent $(g)$ is defined to be ent $\left(g \mid I^{\prime}\right)$, where $I^{\prime}=\bigcap_{j \geq 0} g^{-i}(I)$. Note that $I^{\prime}$ need not be an interval.)

The topological entropy of $g_{n}$ is given by the formula

$$
\text { ent }\left(g_{n}\right)=\log \sigma_{n} \text {, }
$$

† Address for correspondence: Ethan M. Coven, Department of Mathematics, Wesleyan University, Middletown, Conn. 06457, USA. 
where $\sigma_{n}$ is defined as follows. For $k \geq 3$, let $L_{k}(x)=x^{k}-2 x^{k-2}-1$ and let $\lambda_{k}$ denote the largest root of $L_{k}$. For $n=2^{s} k$ where $k$ is odd, let $\sigma_{n}=1$ if $k=1$ and $\sigma_{n}=\left(\lambda_{k}\right)^{2-s}$ if $k \geq 3$.

The result corresponding to Sarkovskii's theorem for maps of the circle is the following [2]: if a continuous map of the circle to itself has a fixed point and a point of least period $n$, then either $(a)$ it has a point of least period $m$ for every $m \triangleright n$ or $(b)$ it has a point of least period $m$ for every $m>n$.

The examples $g_{n}$ of Štefan can be extended to maps of the circle without changing the set of least periods or the topological entropy. L. Block [1] has constructed maps $f_{n}$ of the circle having a fixed point and a point of least period $n$, but no point of least period $m$ for any $m, 1<m<n$. The topological entropy of $f_{n}$ is given by the formula

$$
\text { ent }\left(f_{n}\right)=\log \mu_{n}
$$

where $\mu_{n}$ is the largest root of $M_{n}(x)=x^{n+1}-x^{n}-x-1$.

THEOREM A. If a continuous map of the circle has a fixed point and a point of least period $n \geq 2$, then ent $(f) \geq \min \left\{\log \mu_{n}, \log \sigma_{n}\right\}$.

This result was proved in [3] except for certain maps of degree -1 . See $\S 2$ for details.

In light of the constructions described above, in order to solve the problem stated at the beginning of this paper we need only determine which is smaller, $\mu_{n}$ or $\sigma_{n}$.

THEOREM B. Let $2 \leq n=2^{s} k$ where $k$ is odd.

(1) If $s=0$, then $\mu_{n}<\sigma_{n}$ except that $\mu_{3}=\sigma_{3}$.

(2) If $1 \leq s \leq 6$, then $\sigma_{n}<\mu_{n}$ when $k \leq 2 s+3$ and $\mu_{n}<\sigma_{n}$ when $k \geq 2 s+5$.

(3) If $s \geq 7$, then $\sigma_{n}<\mu_{n}$ when $k \leq 2 s+5$ and $\mu_{n}<\sigma_{n}$ when $k \geq 2 s+7$.

\section{Proof of theorem $A$}

We prove theorem $A$ by restricting our attention to maps of the circle of a fixed degree, denoted by $\operatorname{deg}(\cdot)$, and using the following result.

THEOREM [3]. Let $f$ be a continuous map of the circle having a fixed point and a point of least period $n \geq 2$.

(a) If $|\operatorname{deg}(f)| \geq 2$, then ent $(f) \geq \log |\operatorname{deg}(f)|$.

(b) If $\operatorname{deg}(f)=0$, then ent $(f) \geq \log \sigma_{n}$.

(c) If $\operatorname{deg}(f)=1$, then ent $(f) \geq \min \left\{\log \mu_{n}, \log \sigma_{n}\right\}$.

(d) If $\operatorname{deg}(f)=-1$ and $n$ is odd, then ent $(f) \geq \log \sigma_{n}$.

It is an elementary fact (see $\S 3$ ) that $\mu_{n}, \sigma_{n}<2$. Therefore to prove theorem $A$ it suffices to show that $(d)$ holds when $n$ is even.

LEMMA 1. Let $f$ be a continuous map of the circle having a fixed point and a point of least period $n \geq 3$. If $f$ has no point of least period $n+1$, then ent $(f) \geq \log \sigma_{n}$.

Proof. By theorem $\mathrm{A}_{1}$ of [2], the hypotheses of theorem $\mathrm{A}_{2}$ of [2] are satisfied. Then, as in the proof of theorem $\mathrm{A}_{2}$, there is a proper closed subinterval $K$ of the circle, containing the orbit of a point of least period $n$, a homeomorphism $h$ from 
$K$ onto a compact subinterval $I$ of the reals, and a continuous map $g$ from $I$ into the reals such that for all $x \in K, f(x) \in K$ if and only if $g(h(x)) \in I$, and in this case $h(f(x))=g(h(x))$. In particular, $g$ has a point of least period $n$.

Let $K^{\prime}=\bigcap_{j \geq 0} f^{-i}(K)$ and $I^{\prime}=\bigcap_{j \geq 0} g^{-j}(I)$. Then $f \mid K^{\prime}$ and $g \mid I^{\prime}$ are topologicaily conjugate (via the appropriate restriction of $h$ ) and by definition, ent $(g)=$ ent $\left(g \mid I^{\prime}\right)$. Then

$$
\text { ent }(f) \geq \operatorname{ent}\left(f \mid K^{\prime}\right)=\text { ent }\left(g \mid I^{\prime}\right)=\operatorname{ent}(g) \geq \log \sigma_{n},
$$

the last inequality by Štefan's results.

We now complete the proof of theorem A. Suppose $f$ is a map of the circle of degree -1 having a fixed point and a point of least period $n$, where $n$ is even. We may assume that $n>2$ for, if $n=2$, then $\sigma_{n}=1$ and there is nothing to prove. If $f$ has no point of least period $n+1$, then by lemma 1 , ent $(f) \geq \log \sigma_{n}$. If $f$ has a point of least period $n+1$, then since $n+1$ is odd, ent $(f) \geq \log \sigma_{n+1}$. But it is another elementary fact (see $\S 3$ ) that $\sigma_{m}>\sqrt{2}$ if $m$ is odd, and $\sigma_{m}<\sqrt{2}$ if $m$ is even. Thus $\log \sigma_{n+1}>\log \sigma_{n}$.

\section{Proof of theorem $B$}

We begin by listing some elementary facts about the polynomials

$$
M_{n}(x)=x^{n+1}-x^{n}-x-1 \quad(n \geq 2)
$$

and

$$
L_{k}(x)=x^{k}-2 x^{k-2}-1 \quad(k \geq 3) .
$$

$M_{n}$ is increasing on $(1, \infty)$. Since $M_{n}(1)<0$ and $M_{n}(2)>0, M_{n}$ has a unique root $\mu_{n}$ in $(1, \infty)$ and

$$
\begin{gathered}
1<\mu_{n}<2 . \\
\left(\mu_{n}\right)^{n}=\frac{\mu_{n}+1}{\mu_{n}-1} .
\end{gathered}
$$

$L_{k}^{\prime}$ has exactly one root in $(0, \infty)$ and $L_{k}^{\prime}$ changes from negative to positive at this root. Since $L_{k}(0)<0, L_{k}(\sqrt{2})<0$ and $L_{k}(2)>0, L_{k}$ has a unique root $\lambda_{k}$ in $(0, \infty)$ and

$$
\sqrt{ } 2<\lambda_{k}<2 .
$$

Recall that for $n=2^{s} k$ where $k$ is odd, $\sigma_{n}=1$ if $k=1$ and $\sigma_{n}=\left(\lambda_{k}\right)^{2-s}$ if $k \geq 3$.

$$
\sigma_{n}>\sqrt{2} \text { if } n \text { is odd and } \sigma_{n}<\sqrt{2} \text { if } n \text { is even. }
$$

LEMMA 2. Let $n=2^{s} k$ where $k$ is odd. If $k \geq 2 s+7$, then $\mu_{n}<\sigma_{n}$.

Proof. Let $q=2^{-(s+1)}$. By (3), $\sigma_{n}>2^{q}$. On the other hand,

But

$$
M_{n}\left(2^{q}\right)>0 \text { if } 2^{k / 2}>\frac{2^{q}+1}{2^{q}-1} \text {. }
$$

$$
\frac{2^{x}+1}{2^{x}-1}<\frac{4}{x} \text { whenever } 0<x \leq 1
$$


(To see this, look at $F(x)=(4-x) 2^{x}-x-4 ; F(0)=0$ and $F^{\prime}(x)>0$ if $0 \leq x \leq 1$.) Thus

$$
\frac{2^{a}+1}{2^{q}-1}<2^{s+3}
$$

and hence $M_{n}\left(2^{a}\right)>0$ if $k>2(s+3)$. Therefore

$$
\mu_{2^{s} k}<2^{q}<\sigma_{2^{s} k}
$$

for all odd $k \geq 2 s+7$.

We shall find it desirable to use the polynomials

$$
T_{s}(x)=x^{2^{s+1}}-x-1 \quad(s \geq 0) .
$$

$T_{s}$ is increasing on $(1, \infty)$. Since $T_{s}(1)<0$ and $T_{s}(2)>0, T_{s}$ has a unique root $\tau_{s}$ in $(1, \infty)$ and

$$
\left(\tau_{s}\right)^{2^{s+1}}=\tau_{s}+1
$$

LEMMA 3. Let $n=2^{s} k$ where $k \geq 3$ is odd. Then $\sigma_{n}-\mu_{n}$ has the same sign (positive, negative, zero) as $\left(\tau_{s}+1\right)^{k-2}\left(\tau_{s}-1\right)^{2}-1$.

Proof. Suppose $\sigma_{n}-\mu_{n}>0$. Let $r=2^{s}$. Then $\lambda_{k}>\left(\mu_{n}\right)^{r}$ and hence $L_{k}\left(\left(\mu_{n}\right)^{r}\right)<0$. Writing $\mu$ in place of $\mu_{n}$ and using (2), we have

$$
L_{k}\left(\mu^{r}\right)=\frac{2}{\mu^{2 r}(\mu-1)} T_{s}(\mu) .
$$

Hence $T_{s}(\mu)<0$ and so $\mu<\tau_{s}$. Writing $\tau$ in place of $\tau_{s}$, using (2) and (5) and the fact that $G(x)=((x+1) /(x-1))^{2}$ is decreasing on $(1, \infty)$, we have

$$
\left(\frac{\tau+1}{\tau-1}\right)^{2}<\left(\frac{\mu+1}{\mu-1}\right)^{2}=\mu^{2 n}<\tau^{2 n}=(\tau+1)^{k}
$$

Thus $\left(\tau_{s}+1\right)^{k-2}\left(\tau_{s}-1\right)^{2}>1$.

The same argument goes through with all the inequalities reversed or all replaced by equalities.

An immediate consequence of lemma 3 is

LEMMA 4. Let $k \geq 3$ be odd. If $\mu_{2^{s} k}<\sigma_{2^{s} k}$, then $\mu_{2^{s}}<\sigma_{2^{s}}$ for all odd $l>k$.

LEMMA 5. If $\sigma_{2^{s}(2 s+5)}<\mu_{2^{s}(2 s+5)}$, then $\sigma_{2^{t}(2 t+5)}<\mu_{2^{t}(2 t+5)}$ for all $t>s$.

Proof. $\dagger$ It suffices to show that the result holds for $t=s+1$. Let $\alpha=\tau_{s}$ and $\beta=\tau_{s+1}$. Using (5), we have that $T_{s+1}(\alpha)=\alpha(\alpha+1)>0$, and so $\beta<\alpha$. Using (5) again,

$$
\left(\beta^{2^{s+1}}\right)^{2}=\beta^{2^{s+2}}=\beta+1<\alpha+1=\alpha^{2^{s+1}} \text {. }
$$

Thus $\beta^{2}<\alpha$ and hence $\left(\beta^{2}-1\right)^{2}<(\alpha-1)^{2}$. Then

$$
(\beta+1)^{2(s+1)+3}(\beta-1)^{2}=(\beta+1)^{2 s+3}\left(\beta^{2}-1\right)^{2}<(\alpha+1)^{2 s+3}(\alpha-1)^{2}<1,
$$

the last inequality by lemma 3 . By lemma 3 again, $\sigma_{2^{s+1}(2(s+1)+5)}<\mu_{2^{s+1}(2(s+1)+5)}$.

We now complete the proof of theorem $B$.

Since $M_{3}(x)=\left(x^{2}-x-1\right)\left(x^{2}+1\right)$ and $L_{3}(x)=\left(x^{2}-x-1\right)(x+1), \mu_{3}=\lambda_{3}=\sigma_{3}$. Since $L_{5}(1.5)<0<M_{5}(1.5), \mu_{5}<1.5<\lambda_{5}=\sigma_{5}$. Then (1) follows from lemma 2 .

† This proof is due to M. L. Ginsberg (personal communication). 
It can be checked (using, for example, a hand-held calculator) that if $1 \leq s \leq 6$, then

$$
\sigma_{2^{s}(2 s+3)}<\mu_{2^{s}(2 s+3)}
$$

and

$$
\mu_{2^{s}(2 s+5)}<\sigma_{2^{s}(2 s+5)},
$$

and if $s=7$, then

$$
\sigma_{2^{s}(2 s+5)}<\mu_{2^{s}(2 s+5)} .
$$

Then (2) follows from lemma 4 and (3) follows from lemmas 2, 4 and 5 .

\section{REFERENCES}

[1] L. Block. Periodic orbits of continuous maps of the circle. Trans. Amer. Math. Soc. 260 (1980), $555-562$.

[2] L. Block. Periods of periodic points of maps of the circle which have a fixed point. Proc. Amer. Math. Soc. 82 (1981), 481-486.

[3] L. Block, J. Guckenheimer, M. Misiurewicz, \& L.-S. Young. Periodic points and topological entropy of one dimensional maps. In Global Theory of Dynamical Systems, Proceedings, pp. 18-34. Northwestern, 1979. Lecture Notes in Math. no. 819. Springer: Berlin, 1980.

[4] A. N. Sarkovskii. Co-existence of cycles of a continuous mapping of the line into itself. Ukrain. Mat. Ž. 16 (1964) 61-71. (Russian, English summary.)

[5] P. Štefan. A theorem of Šarkovskii on the existence of periodic orbits of continuous endomorphisms of the real line. Comm. Math. Phys. 54 (1977), 237-248. 